\title{
SÍNTESE E CARACTERIZAÇÃO DE ÓXIDOS MISTOS V/Mn-Co-
} Al A PARTIR DE PRECURSORES TIPO HIDROTALCITA E AVALIAÇÃO CATALÍTICA NA REAÇÃO DE DECOMPOSIÇÃO DO ISOPROPANOL

\author{
G.U. DOS SANTOS ${ }^{1}$, S. OKAMOTO ${ }^{2}$ e G.G. CORTEZ ${ }^{1}$ \\ ${ }^{1}$ Escola de Engenharia de Lorena, Universidade de São Paulo, Departamento de Engenharia \\ Química \\ ${ }^{2}$ Laboratório Associado de Combustão e Propulsão, Instituto Nacional de Pesquisas Espaciais \\ E-mail para contato: cortez@ dequi.eel.usp.br; gabiuchona@gmail.com; sayuri@1cp.inpe.br
}

\begin{abstract}
RESUMO - Os catalisadores à base de vanádio têm recebido muita atenção nos últimos anos devida a sua elevada atividade e seletividade em reações de grande importância industrial, tais como a transformação de hidrocarbonetos em produtos químicos de comprovada aplicação industrial. Embora o comportamento catalítico destes catalisadores seja bem conhecido pelos pesquisadores, muito se tem discutido sobre a natureza de seus sítios ativos, sua interação com o suporte e com as espécies químicas a serem catalisadas. Em vista disso, foi utilizado em nosso trabalho a reação de decomposição do isopropanol para a investigação de caráter ácido e básico de sistema VOx/Mn-Co-Al. Neste contexto, o principal objetivo deste estudo foi avaliar a influência da presença do vanádio sobre um óxido misto $\mathrm{Mn}-\mathrm{Co}-\mathrm{Al}$ cujo precursor seja uma hidrotalcita preparada com diferentes concentrações de manganês, cobalto e alumínio. Os catalisadores foram caracterizados por diferentes técnicas, tais como área superficial, redução à temperatura programada e difratometria de raios X. A atividade catalítica e as propriedades ácidas e/ou básicas dos catalisadores foram avaliadas através da reação de decomposição do isopropanol.
\end{abstract}

\section{INTRODUÇÃO}

A hidrotalcita é a argila aniônica natural mais conhecida e é composta por ânions carbonato intercalados entre lamelas de hidróxido duplo de magnésio e alumínio. Esses compostos são os precursores mais promissores de catalisadores multicomponentes para muitas reações catalíticas de interesse industrial. Alguns relatos na literatura mostram que derivados de óxidos de materiais do tipo hidrotalcitas têm qualidades interessantes para a área de catálise, como, por exemplo, uma boa distribuição dos metais no sólido (Corrêa et al., 2016).

Estes óxidos mistos são potencialmente utilizados como catalisadores por apresentarem uma elevada área específica, estabilidade térmica e distribuição homogênea dos cátions inseridos na estrutura (Carja et al., 2003). 
A cada dia vem crescendo o interesse pelos estudos de catalisadores de óxidos mistos em busca de melhores rendimentos e seletividades. Alguns dos óxidos como óxidos de nióbio, vanádio e alumínio vêm sendo estudados buscando melhor desempenho catalítico. Catalisadores de vanádio suportado, por exemplo, vêm sendo utilizados em um grande número de reações de oxidação seletiva (Paulino et al., 2007).

Algumas características são importantes quando se leva em conta o óxido suporte utilizado, tais como elevada área superficial, estabilidade térmica sob determinadas condições reacionais e propriedades físico-químicas necessárias para uma alta atividade das espécies ativas durante a reação (Cherian et al., 2003). Por conta desses aspectos, o objetivo do trabalho foi preparar catalisadores do tipo $\mathrm{VO}_{\mathrm{x}} / \mathrm{Mn}-\mathrm{Co}-\mathrm{Al}$, caracterizá-los a partir das técnicas de difratometria de raios $\mathrm{X}(\mathrm{DRX})$, volumetria de $\mathrm{N}_{2}$ e redução à temperatura programada (TPR) e avaliar suas propriedades ácidas e/ou básicas pela reação de decomposição do isopropanol. O suporte, à base de manganês, cobalto e alumínio, foi escolhido levando em consideração alguns estudos realizados, como por exemplo, sua excelente atividade na oxidação de CO e hidrocarbonetos (Sim et al., 2014).

\section{METODOLOGIA}

\subsection{Síntese dos catalisadores}

Em um reator batelada de $1 \mathrm{~L}$ de capacidade, sob aquecimento com banho termostático a $40^{\circ} \mathrm{C}$ e agitação, foi adicionado $150 \mathrm{~mL}$ de $\mathrm{Na}_{2} \mathrm{CO}_{3}(1 \mathrm{M})$. Com o auxílio de duas bombas peristálticas, adicionaram-se $200 \mathrm{~mL}$ de solução contendo $\mathrm{Al}\left(\mathrm{NO}_{3}\right)_{3} .9 \mathrm{H}_{2} \mathrm{O}, \mathrm{Co}\left(\mathrm{NO}_{3}\right) 2.6 \mathrm{H}_{2} \mathrm{O}$ e $\mathrm{Mn}\left(\mathrm{NO}_{3}\right)_{2} \cdot 4 \mathrm{H}_{2} \mathrm{O}(0,5 \mathrm{M})$ e $250 \mathrm{~mL}$ de $\mathrm{NaOH}(1 \mathrm{M})$, mantendo o $\mathrm{pH}$ em 10 durante toda a reação. $\mathrm{O}$ produto permaneceu sob envelhecimento sob agitação a $40^{\circ} \mathrm{C}$ por 16 horas, após o qual foi lavado e filtrado a vácuo, até a água de lavagem atingir $\mathrm{pH}$ 7. O produto foi seco a $110^{\circ} \mathrm{C}$ por 24 horas, triturado e selecionado granulometricamente em peneira com malha de abertura $0,150 \mathrm{~mm}$. $\mathrm{O}$ material foi calcinado em uma mufla à temperatura de $450^{\circ} \mathrm{C}$ por 5 horas. Foram sintetizados três tipos de precursores: 1Mn1Co38Al, 1,5Mn0,5Co38Al e $0,5 \mathrm{Mn} 1,5 \mathrm{Co} 38 \mathrm{Al}$.

Os suportes foram impregnados com vanádio a partir de duas séries. A primeira série, impregnada com uma monocamada de 8 átomos de vanádio $/ \mathrm{nm}^{2}$, foi realizada através da mistura da solução de $\mathrm{NH}_{4} \mathrm{VO}_{3}$, aquecida a $70^{\circ} \mathrm{C}$, e o suporte na forma de pó em um rotoevaporador a vácuo. Após essa etapa, o pó residual foi seco a $110^{\circ} \mathrm{C}$ por $24 \mathrm{~h}$ na estufa, e depois calcinado a $450^{\circ} \mathrm{C}$ por $5 \mathrm{~h}$ na mufla, obtendo-se assim o catalisador identificado como $1 \mathrm{~V} / \mathrm{MnCoAl}$. A segunda série, impregnada com meia monocamada de 4 átomos de vanádio/nm ${ }^{2}$, foi obtida pela mesma rota citada, obtendo-se o catalisador $0,5 \mathrm{~V} / \mathrm{MnCoAl}$.

\subsection{Caracterização físico-química}

As análises de difração de raios $\mathrm{X}$ foram realizadas a partir do método do pó para identificação de estruturas e fases cristalinas presentes (Chenari et al., 2016). A fonte utilizada foi de $\mathrm{CuK} \alpha \mathrm{em}$ um intervalo de varredura de $2 \theta$, variando de 5 a $80^{\circ}$, a uma velocidade angular do goniômetro de $0,02 \%$, em um difratômetro X'Pert PRO da PANalytical. As fases cristalinas foram identificadas com auxílio do programa graph analyzer criado pelo INPE de Cachoeira Paulista. 
As medidas de áreas específicas, volume de poros (método BET) e distribuição de volume de poros (método $\mathrm{BJH}$ ) foram determinadas a partir das isotermas de adsorçãodessorção de $\mathrm{N}_{2}$ a $-196^{\circ} \mathrm{C}$, utilizando-se um equipamento da marca Quantachrome, modelo NOVA 1000. As amostras foram previamente tratadas in situ sob vácuo e aquecimento a $100^{\circ} \mathrm{C}$, por $2 \mathrm{~h}$.

As análises de redução à temperatura programada (RTP) dos catalisadores suportados foram realizadas em um equipamento Quantachrome, modelo Chembet-3000, equipado com um detector de condutividade térmica. As amostras $(0,04 \mathrm{~g})$ foram secas in situ a $110{ }^{\circ} \mathrm{C}$ por $1 \mathrm{~h}$ sob fluxo de hélio. Em seguida, os sólidos foram resfriados à temperatura ambiente e reduzidos até $900{ }^{\circ} \mathrm{C}\left(10{ }^{\circ} \mathrm{C} / \mathrm{min}\right)$ com uma mistura de $5 \% \mathrm{H}_{2} / \mathrm{N}_{2}(30 \mathrm{~mL} / \mathrm{min})$.

Os catalisadores foram avaliados na reação de decomposição do isopropanol, empregando-se um reator de leito fixo (borosilicato) com fluxo contínuo dos reagentes sob pressão atmosférica. O isopropanol $(99,7 \%)$ foi injetado na tubulação de alimentação de gases afluentes do reator com auxílio de uma bomba Thermo Separation Products, modelo Spectra P100, na vazão de $0,02 \mathrm{mLmin}^{-1}$ e diluído em He $\left(37 \mathrm{mLmin}^{-1}\right)$. Os gases efluentes do reator foram analisados por cromatografia gasosa, utilizando-se um cromatógrafo da marca Varian, modelo 3380, equipado com um DCT, utilizando uma coluna empacotada com fase $20 \% \mathrm{CW}$ 20M (80/100 mesh) com $4 \mathrm{~m}$ de comprimento, $2 \mathrm{~mm}$ DI, 1/8" DN. A reação foi avaliada no intervalo de temperatura de $240^{\circ} \mathrm{C}$ e $320^{\circ} \mathrm{C}$, em reator tubular de leito fixo contendo $150 \mathrm{mg}$ do material catalítico e um fator de tempo de residência $\left(\mathrm{W} / \mathrm{F}_{\mathrm{A} 0}\right)$ igual a $9,5 \mathrm{~g} \cdot \mathrm{h} / \mathrm{mol}_{\text {Isop. }}$. A conversão do isopropanol foi calculada a partir do balanço da quantidade de carbono, assumindo como sendo constante o balanço de carbono presente na saída do reator e no fluxo de alimentação do isopropanol. A partir disso, a conversão do reagente $\left(\mathrm{X}_{\text {Isop }}\right)$, a atividade do catalisador $(\mathrm{r})$, a velocidade de formação $\left(\mathrm{VFP}_{\mathrm{i}}\right)$ e o rendimento dos produtos $\left(\mathrm{R}_{\mathrm{i}}\right)$ foram calculados pelas seguintes equações:

$$
\begin{aligned}
& \mathrm{X}_{\text {isop }}(\%)=\left(1-\mathrm{n}_{\mathrm{s}} / \mathrm{n}_{\mathrm{e}}\right) * 100 \\
& \mathrm{r}=\left(\mathrm{F}_{\text {isop }(\mathrm{e})} * \mathrm{X}_{\text {isop }}\right) /(\mathrm{M} * 100) \\
& \mathrm{VFP}_{\mathrm{i}}=\left(\mathrm{S}_{\mathrm{i}} * \mathrm{r}\right) / 100 \\
& \quad \text { onde } \mathrm{S}_{\mathrm{i}}(\%)=\left(n i / \sum_{\mathrm{i}=1}^{\mathrm{n}} n i\right) * 100 \\
& \mathrm{R}_{\mathrm{i}}(\%)=\mathrm{X}_{\text {isop }} * \mathrm{~S}_{\mathrm{i}}
\end{aligned}
$$

onde $n_{\mathrm{e}}$ e $n_{\mathrm{s}}$ são, respectivamente, o número de mol de isopropanol na entrada e saída do reator, $\mathrm{F}_{\mathrm{isop(e)}}$ é o fluxo de isopropanol na entrada do reator, $\mathrm{M}$ é a massa do catalisador, $\mathrm{S}_{\mathrm{i}}$ é a seletividade do produto i e $\mathrm{n}_{\mathrm{i}}$ é a quantidade de matéria do produto i no efluente do reator.

\section{RESULTADOS E DISCUSSÕES}

A tabela 1 mostra os valores obtidos de área superficial específica $\left(\mathrm{S}_{\mathrm{BET}}\right)$, volume de poros $\left(\mathrm{V}_{\mathrm{P}}\right)$ e diâmetro médio de poros $\left(\mathrm{D}_{\mathrm{P}}\right)$ para os suportes de $\mathrm{Mn}-\mathrm{Co}-\mathrm{Al}$ e para os catalisadores à base de vanádio. 
Tabela 1 - Valores de área específica $\left(\mathrm{S}_{\mathrm{BET}}\right)$, volume de poros $\left(\mathrm{V}_{\mathrm{P}}\right)$ e diâmetro médio de poros $\left(\mathrm{D}_{\mathrm{P}}\right)$ dos catalisadores

\begin{tabular}{|c|c|c|c|}
\hline Amostras & $\mathrm{S}_{\mathrm{BET}}$ & $\mathrm{V}_{\mathrm{P}}$ & $\mathrm{D}_{\mathrm{P}}$ \\
\hline $1 \mathrm{Mn} 1 \mathrm{Co} 38 \mathrm{Al}$ & 252 & 0,55 & 3,0 \\
\hline $0,5 \mathrm{Mn} 1,5 \mathrm{Co} 38 \mathrm{Al}$ & 367 & 0,61 & 2,2 \\
\hline $1,5 \mathrm{Mn} 0,5 \mathrm{Co} 38 \mathrm{Al}$ & 297 & 0,60 & 2,8 \\
\hline $0,5 \mathrm{~V} / 1 \mathrm{Mn} 1 \mathrm{Co} 38 \mathrm{Al}$ & 162 & 0,42 & 3,0 \\
\hline $0,5 \mathrm{~V} / 0,5 \mathrm{Mn} 1,5 \mathrm{Co} 38 \mathrm{Al}$ & 152 & 0,28 & 2,7 \\
\hline $0,5 \mathrm{~V} / 1,5 \mathrm{Mn} 0,5 \mathrm{Co} 38 \mathrm{Al}$ & 122 & 0,28 & 3,0 \\
\hline $1 \mathrm{~V} / 1 \mathrm{Mn} 1 \mathrm{Co} 38 \mathrm{Al}$ & 93 & 0,28 & 3,0 \\
\hline $1 \mathrm{~V} / 0,5 \mathrm{Mn} 1,5 \mathrm{Co} 38 \mathrm{Al}$ & 110 & 0,24 & 2,7 \\
\hline $1 \mathrm{~V} / 1,5 \mathrm{Mn} 0,5 \mathrm{Co} 38 \mathrm{Al}$ & 101 & 0,24 & 6,0 \\
\hline
\end{tabular}

Pode-se observar que, em relação aos óxidos mistos calcinados, a variação nas proporções de $\mathrm{Mn}$ e Co levou a um aumento mais significativo na área superficial para o composto $0,5 \mathrm{Mn} 1,5 \mathrm{Co} 38 \mathrm{Al}$. A introdução de vanádio no óxido misto também provocou alterações texturais na sua estrutura, o que pode ser observado pela variação no volume dos poros e a diminuição da área específica na série $0,5 \mathrm{~V} / \mathrm{MnCoAl}$. Com o aumento de vanádio sobre o óxido misto, observou-se algumas alterações nas áreas específicas e volume de poros que podem estar relacionadas ao bloqueio dos poros devido ao aumento da concentração de vanádio durante o processo de impregnação do sal de vanádio na série $1 \mathrm{~V} / \mathrm{MnCoAl}$.

As Figuras 1 e 2 apresentam a isoterma de adorção-dessorção de $\mathrm{N}_{2}$ e distribuição de tamanho de poros, respectivamente, dos catalisadores. Pela Figura 1, percebe-se que os catalisadores se enquadram na isoterma do tipo IV e possui histerese do tipo H2 (Figueiredo, J.L.; Ribeiro, F.R., 1989). A curva inferior da isoterma de adsorção mostra a quantidade de gás adsorvida com o aumento da pressão relativa, enquanto que o ramo superior representa a quantidade de gás dessorvida no processo inverso. Este tipo de isoterma é característica de sólidos mesoporosos (2-50 nm), nos quais ocorre o fenômeno de condensação capilar em que a quantidade adsorvida tende para um valor máximo finito, correspondente ao enchimento completo dos capilares com adsorvido no estado líquido.

Na Figura 2, encontra-se a curva de distribuição de tamanho de poros dos catalisadores, construída a partir da derivação da curva de volume acumulado. O fenômeno de condensação capilar também pode ser utilizado na determinação da distribuição do tamanho de poros na faixa mesoporosa (Teixeira et al., 2001). Podemos observar que a maior parte dos poros dos catalisadores apresenta diâmetro máximo entre 2,0-6,0 nm, caracterizando-os como materiais mesoporosos, como citado acima. 
Figura 1 - Isotermas de adsorção-dessorção de $\mathrm{N}_{2}$ dos catalisadores
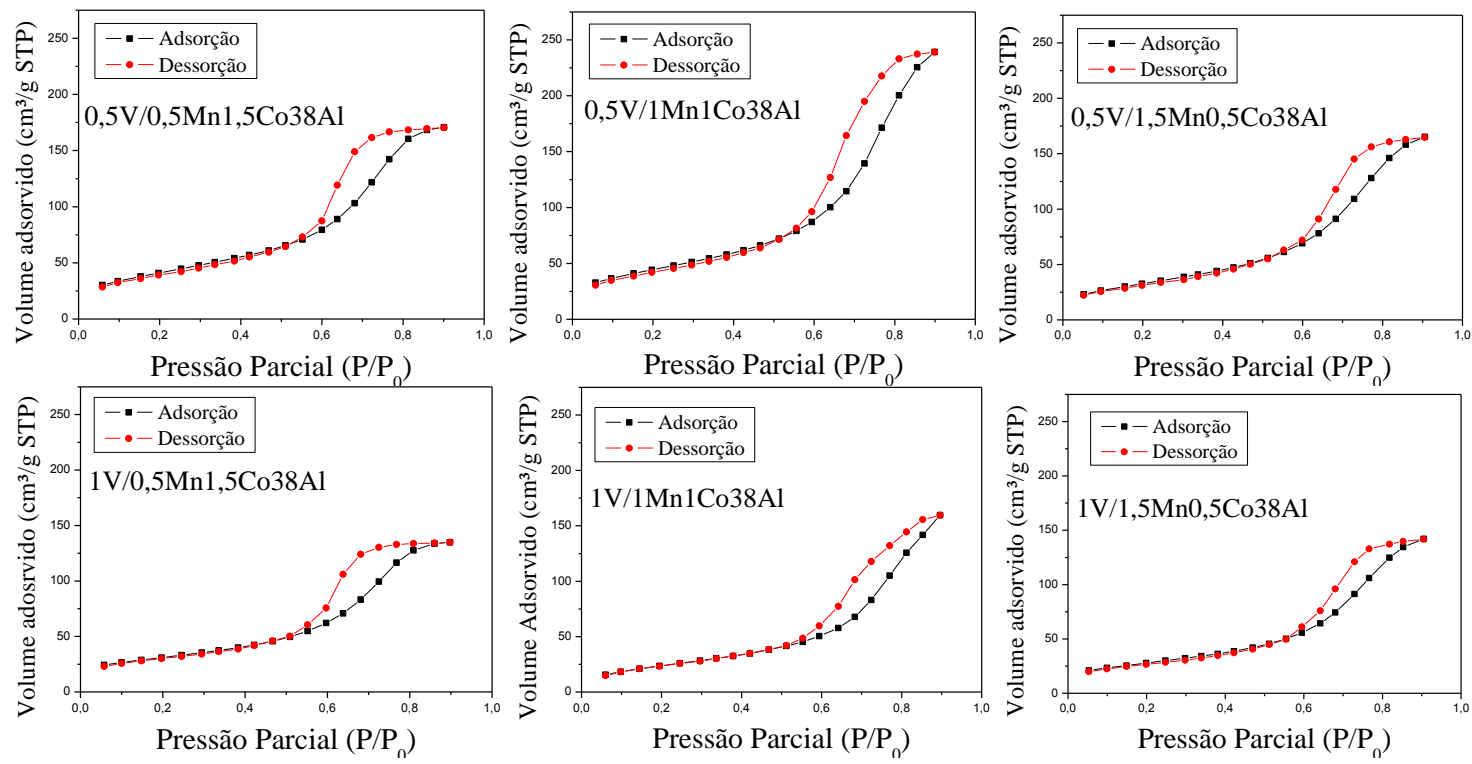

Figura 2 - Distribuição de tamanho de poros dos catalisadores
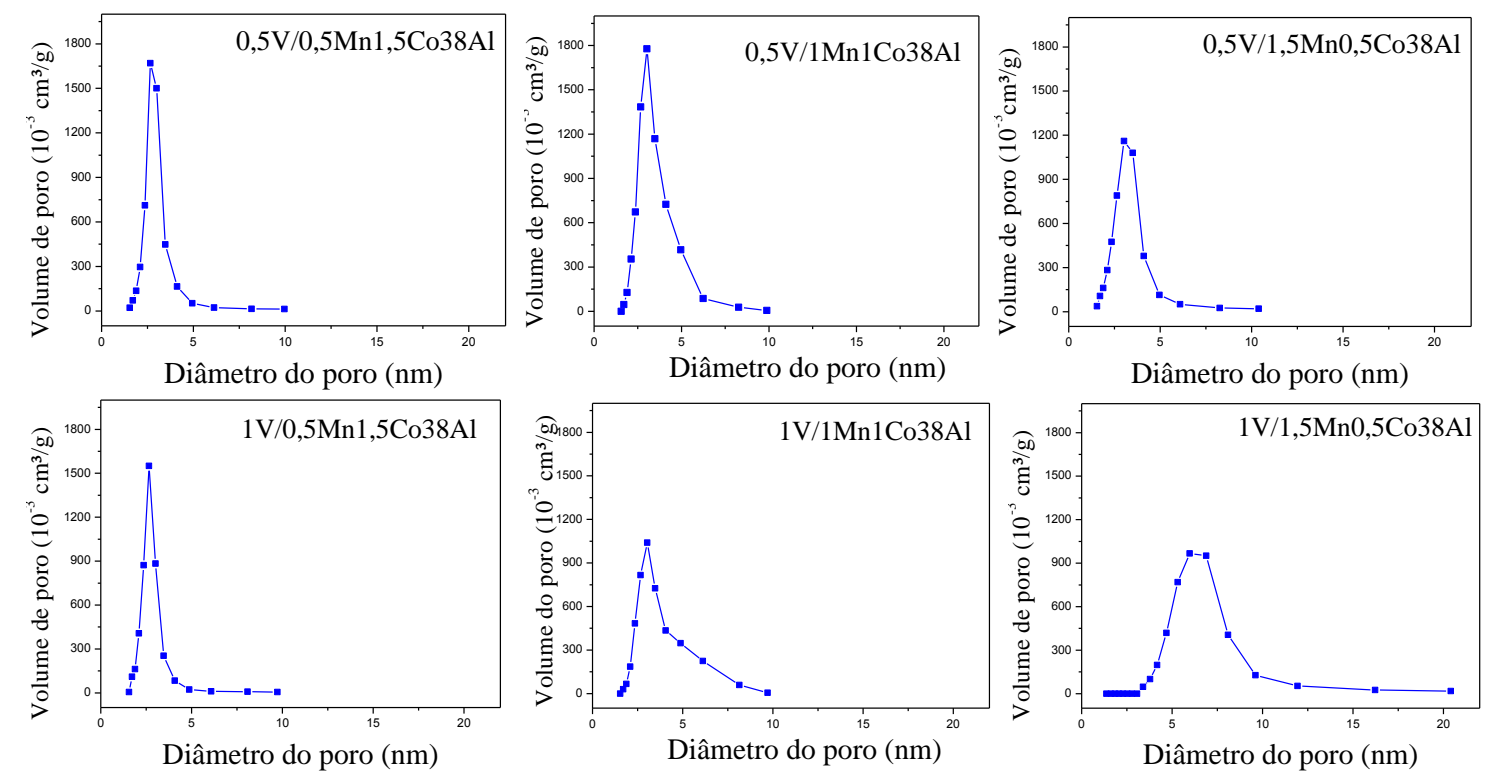

A Figura 3 representa a análise de difração de raios $X$ (DRX) dos precursores secos MnCoAl a fim de se verificar a existência da fase hidrotalcita. Comparando-a com o difratograma encontrado na literatura (Li et al., 2015), pôde-se observar a presença da fase hidrotalcita nos precursores. 
Figura 3 - Difratograma de raios $\mathrm{X}$ dos precursores

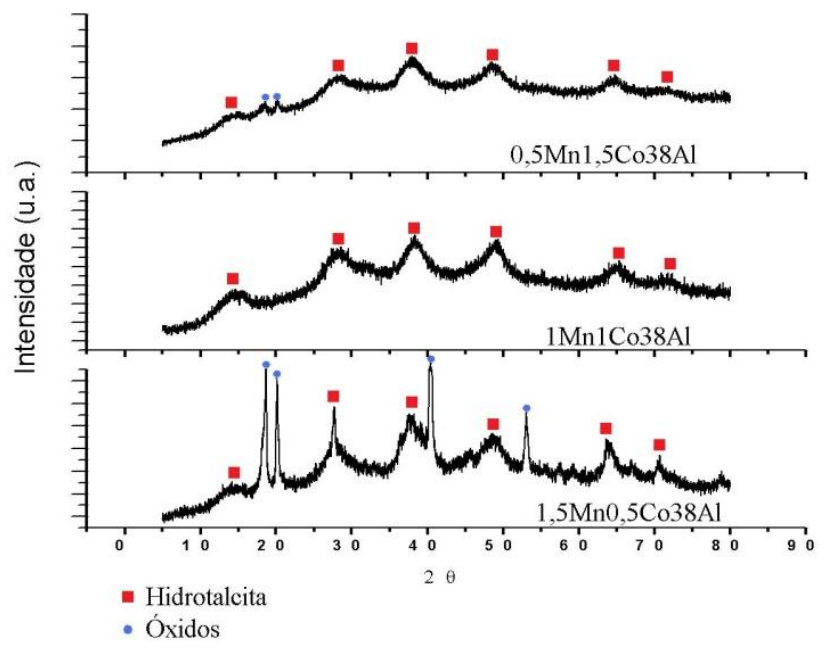

As análises de difratometria de raios $\mathrm{X}$ para os catalisadores sintetizados e calcinados estão representadas na Figura 4. Uma das maneiras de identificar o produto é comparar as fichas ICDD existentes com o difratograma obtido.

Figura 4 - DRX das amostras. Picos referentes ao Mn (־), ao Co (־) e ao $\mathrm{Al}(())$.

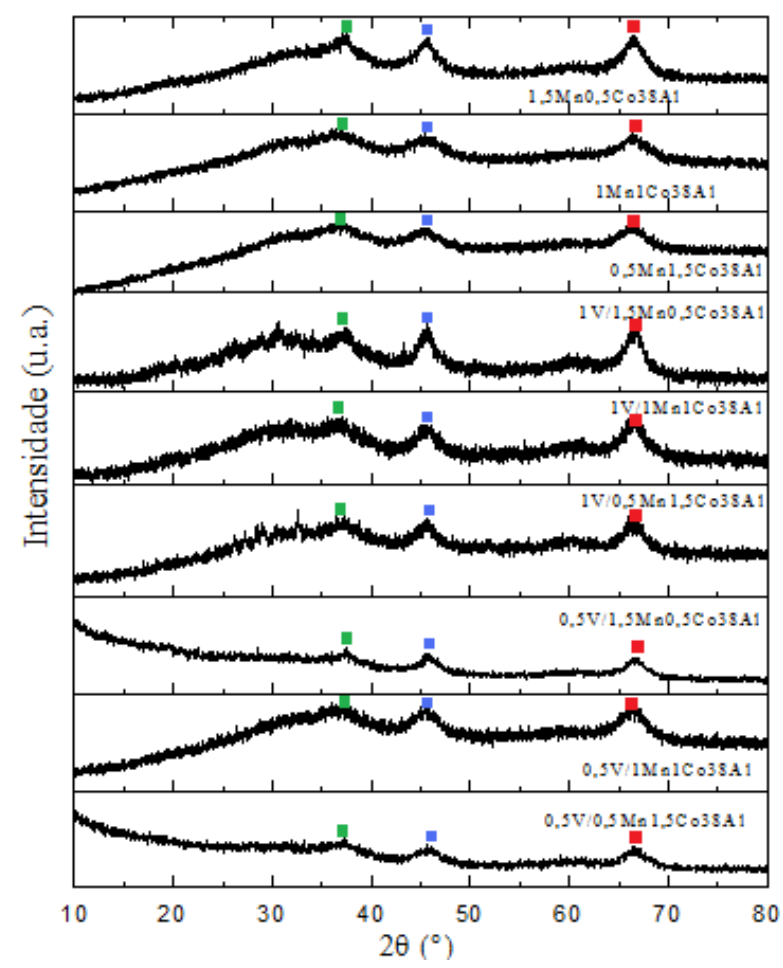

A partir dessas análises de DRX, pôde-se perceber a formação de espinélio para todas as amostras. Isso ocorre devido à característica amorfa do material, observada pelo alargamento dos picos e causada pela presença do cobalto na estrutura (Castaño et al., 2013). Como se pode observar nos DRX, as amostras que contém maior quantidade de manganês na estrutura 
apresentaram picos melhores definidos (Castaño et al., 2013). No pico correspondente a $2 \theta$ em torno de $37^{\circ}$ está presente a fase de espinélio contendo manganês na sua estrutura, tanto na sua fase de óxido $\left(\mathrm{MnAl}_{2} \mathrm{O}_{4}\right)$ quanto na presença do vanádio, $\mathrm{Mn}_{4} \mathrm{~V}_{2} \mathrm{O}_{9}$ (ICDD 00-0011302 e 00-031-0848). O pico para $2 \theta$ igual a $45^{\circ}$ corresponde à fase de espinélio contendo cobalto na estrutura, onde aparece $\mathrm{CoAl}_{2} \mathrm{O}_{4}$ e $\mathrm{Co}\left(\mathrm{VO}_{3}\right)_{2}$ (ICDD 00-003-0896 e 00-051-0024). Para $2 \theta$ em torno de $66^{\circ}$, encontram-se os picos referentes aos compostos que possuem $\mathrm{Al}$ na sua estrutura, como $\mathrm{Al}(\mathrm{OH})_{3}$ e $\mathrm{AlV}_{2} \mathrm{O}_{4}$ (ICDD 01-070-2038 e 00-025-0025). Percebe-se que os espinélios formados se apresentaram a partir de uma mistura dos elementos existentes na composição e não como óxidos puros de cada elemento.

Segundo um trabalho publicado por Nieto et al. (1995), as hidrotalcitas são estáveis quando submetidas a temperatura de calcinação de até a $400^{\circ} \mathrm{C}$ e acima de $500^{\circ} \mathrm{C}$, estes se transformam em óxidos de $\mathrm{Al}$ que rehidrata pela impregnação com a solução aquosa, regenerando a sua estrutura. Contudo, analisando os DRX dos catalisadores impregnados, pôde-se afirmar que isso ocorreu de forma bem sutil.

Pelos resultados de DRX dos catalisadores, verificou-se a presença dos óxidos de vanádio na estrutura, porém não com sua forma pura $\left(\mathrm{V}_{2} \mathrm{O}_{5}\right)$, mas sim interagida com os elementos presentes nos seus suportes.

As análises de RTP dos suportes e dos catalisadores estão representadas pelas Figuras 5 e 6, respectivamente.

Figura 5 - RTP dos suportes.

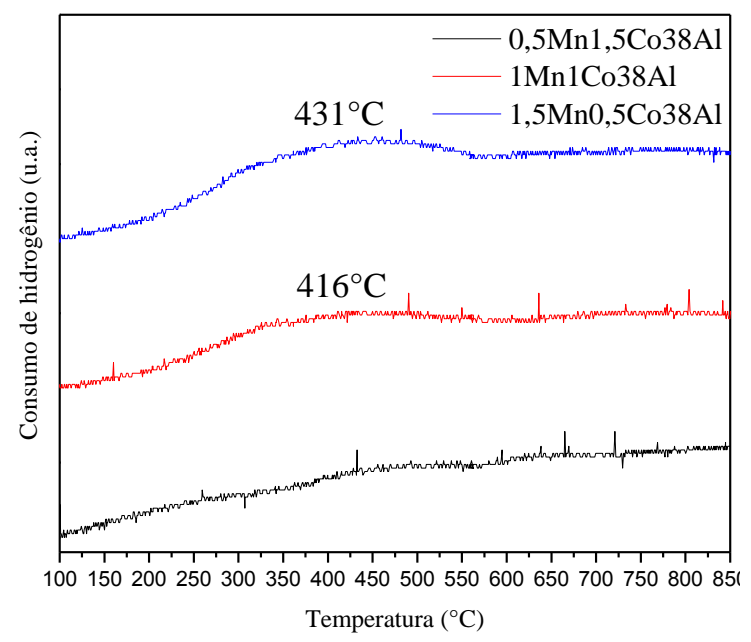

Pelos resultados de RTP dos suportes, verifica-se que para baixa concentração de manganês, não houve o aparecimento de nenhum pico de redução. À medida que foi aumentando a quantidade de $\mathrm{Mn}$ na estrutura, podemos observar o aparecimento de um ombro crescente na faixa de temperatura desde $416^{\circ} \mathrm{C}$ para o suporte $1 \mathrm{Mn} 1 \mathrm{Co} 38 \mathrm{Al}$ e $431^{\circ} \mathrm{C}$ para $1,5 \mathrm{Mn} 0,5 \mathrm{Co} 38 \mathrm{Al}$. Tanto os óxidos de $\mathrm{Mn}$ e como o de $\mathrm{Co}$, se reduzem a uma temperatura em torno de $450^{\circ} \mathrm{C}$, sendo que o $\mathrm{Mn}$ reduz do estado $\mathrm{Mn}_{3} \mathrm{O}_{4}$ para $\mathrm{MnO}$; e o $\mathrm{Co}$, do $\mathrm{Co}_{3} \mathrm{O}_{4}$ para $\mathrm{CoO}$ (Lamonier et al., 2007). A difícil visualização se deve a superposição dos ombros de redução dos compostos. 
Figura 6 - RTP dos catalisadores.

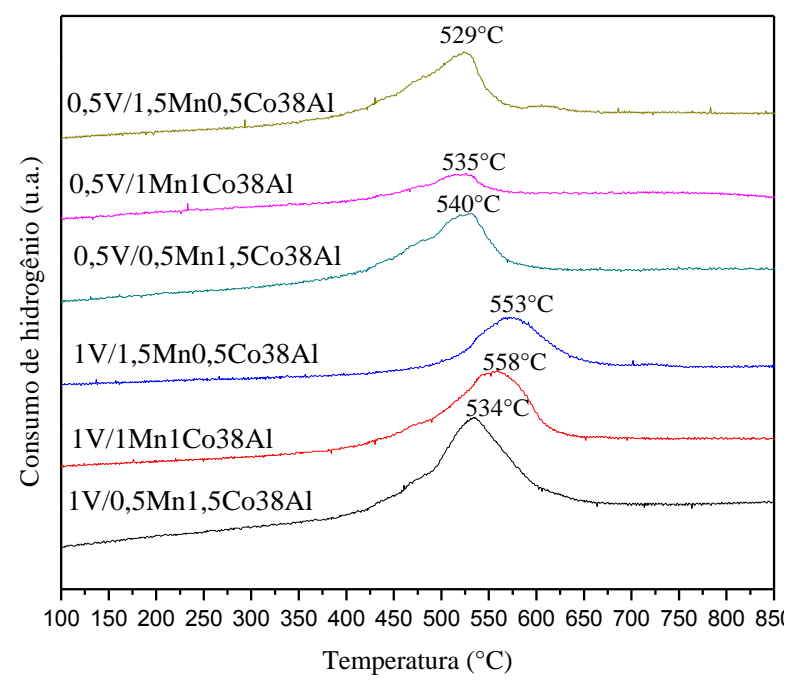

Os catalisadores de vanádio suportado sobre os óxidos mistos MnCoAl exibiram um único pico entre 529 e $560^{\circ} \mathrm{C}$ e ele é correspondente à redução de $\mathrm{V}_{2} \mathrm{O}_{5}$ a $\mathrm{V}_{6} \mathrm{O}_{13}$. Geralmente, $\mathrm{V}_{2} \mathrm{O}_{5}$ mássico apresenta picos de redução em temperaturas iguais a 655,686 e $807^{\circ} \mathrm{C}$ (Arfaoui et al., 2016) e a ausência desses picos, neste caso, resulta de uma alta dispersão do vanádio sobre o óxido misto MnCoAl. Pode-se observar também que, em uma das séries, aumentandose a proporção de Mn no precursor, a temperatura de redução diminui.

Figura 7 - Testes catalíticos das espécies V/MnCoAl
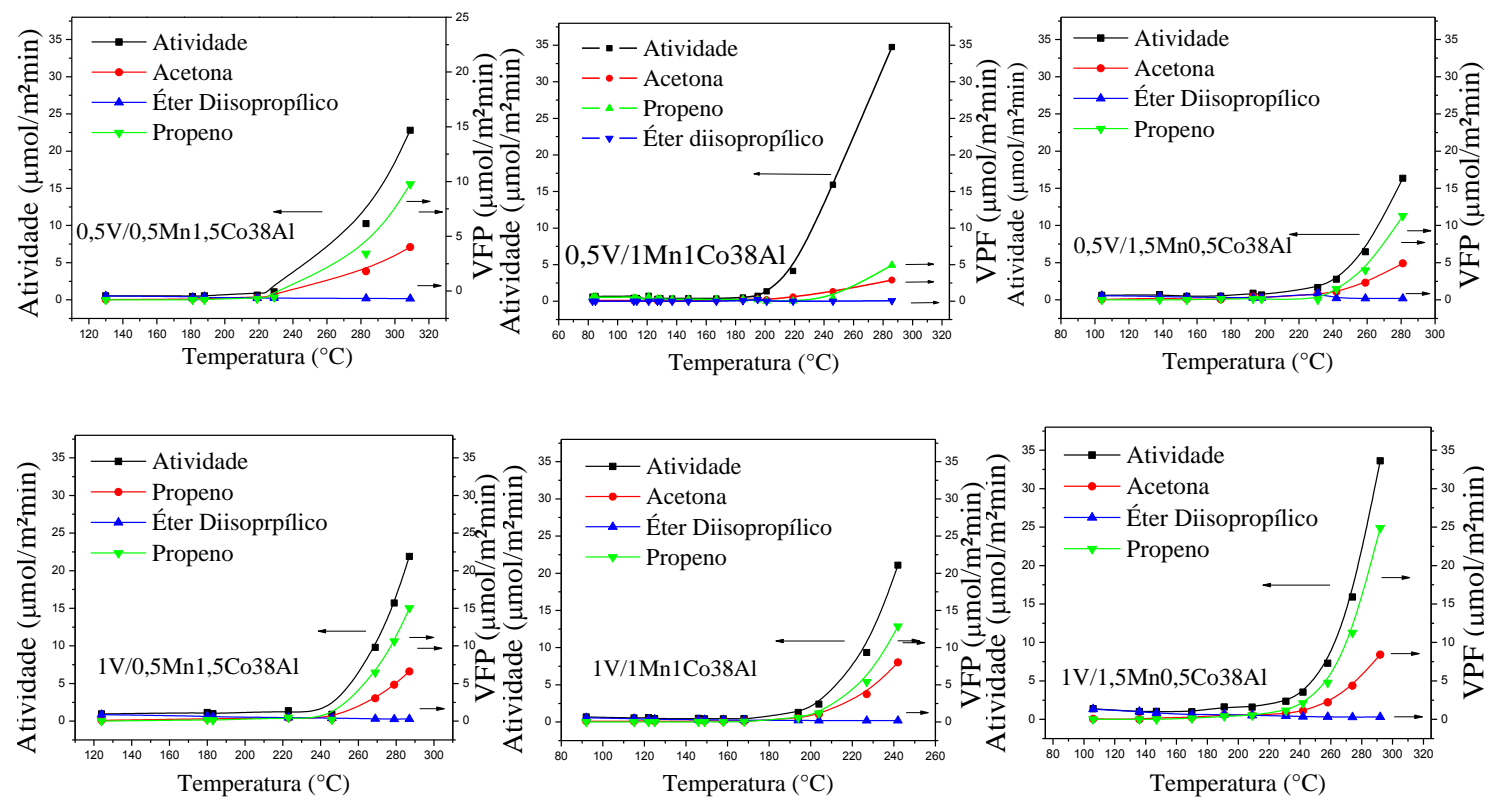

Nos resultados de avaliação catalítica na decomposição do isopropanol, o óxido misto $\mathrm{MnCoAl}$, com diferentes composições de $\mathrm{Mn}$ e Co, mostrou-se praticamente inativo na formação dos produtos a altas temperaturas. A partir disso, foram realizados os testes 
catalíticos dos catalisadores impregnados com vanádio representados pela Figura 7. Verificase uma atividade catalítica bem elevada devido à presença do vanádio na estrutura e observase que a formação dos produtos se inicia entre temperaturas de 210 e $240^{\circ} \mathrm{C}$. A decomposição do isopropanol por meio de sítios básicos se procede através de uma reação de eliminação produzindo acetona. Em meios ácidos, o isopropanol se desidrata formando propileno e éterisopropílico (7). Sabendo que a reação de decomposição do isopropanol forma produtos de desidrogenação (acetona) e desidratação (propeno e éter diisopropílico), devido aos sítios de diferentes naturezas (básico e ácido, respectivamente), pode-se afirmar que os catalisadores apresentam sítios ácidos e básicos, evidenciado pela formação do propeno e acetona como produtos finais. Fixando a avaliação na temperatura de $280^{\circ} \mathrm{C}$, pode-se concluir que, para a série $0,5 \mathrm{~V}$, o catalisador obtido pela impregnação sobre o $1,5 \mathrm{Mn} 0,5 \mathrm{Co} 38 \mathrm{Al}$ apresentou melhor atividade para conversão do isopropanol para acetona e para propeno. Já para a série $1 \mathrm{~V}$, todos os catalisadores apresentaram resultados satisfatórios e semelhantes em relação a conversão para propeno e acetona. Nenhum dos catalisadores produziu éter diisopropílico.

\section{CONCLUSÃO}

Os três precursores sintetizados pelo método da coprecipitação apresentaram a formação de composto do tipo hidrotalcita. Entretanto, mesmo após a calcinação e a formação de espinélios na estrutura, pôde-se observar, posteriormente, a formação de catalisadores de óxidos mistos de V/MnCoAl, comprovado pelas análises de DRX. Com a análise de RTP, concluiu-se que o houve uma dispersão característica do vanádio sobre o óxido misto MnCoAl. A partir da avaliação catalítica, foi possível observar também que a impregnação com vanádio gerou um aumento na atividade catalítica e a produção de propeno e acetona se deve às propriedades redox do vanádio na superfície do óxido misto. Dentre os catalisadores das séries $0,5 \mathrm{~V}$ e $1 \mathrm{~V}$, pode-se concluir que o catalisador $1 \mathrm{~V} / 1,5 \mathrm{Mn} 0,5 \mathrm{Co} 38 \mathrm{Al}$ foi o mais efetivo, pois observa-se que, para uma mesma temperatura, em torno de $280^{\circ} \mathrm{C}$, ele foi o qual apresentou a maior conversão para propeno.

\section{AGRADECIMENTOS}

Ao LABCP-INPE de Cachoeira Paulista/SP e à FAPESP (2015/19391-5).

\section{REFERÊNCIAS}

ARFAOUI, J.; GHORBEL, A.; PETITTO, C.; DELAHAY, G. Novel Vanadium supported onto mixed Molybdenum-Titanium Pillared Clay catalysts for the low temperature SCR-NO by $\mathrm{NH}_{3}$. Chemical Engineering Journal, 2017.

CARJA, G.; NAKAMURA, R.; AINDA, T.; NIIYAMA, H. Mg-V-Al mixed oxides with mesoporous properties using layered double hydroxides as precursors: catalytic behavior for the process of ethylbenzene dehydrogenation to styrene under a carbon dioxide flow. Journal of Catalysis, v. 218, p. 104-110, 2003.

CASTAÑO, M.H.; MOLINA, R.; MORENO, S. Mn-Co-Al-Mg mixed oxides by autocombustion method and their use as catalysts in the total oxidation of toluene. Journal of Molecular Catalysis A: Chemical, v. 370, p. 167-174, 2013. 
CHENARI, H.M.; SÁNCHEZ-BAJO, F. Microstructural study of $\mathrm{SnO}_{2}$-based nanoparticles by X-ray diffractometry. Materials Science in Semiconductor Processing, v. 49, p. 15-19, 2016.

CHERIAN, M.; RAO, M.S.; DEO, G. Niobium oxide as support material for the oxidative dehydrogenation of propane. Catalysis Today, v. 78, p. 397-409, 2003.

CORRÊA, C.L.O.; LICEA, Y.E.; PALACIO, L.A.; ZOTIN, F.M.Z.. Effect of composition and thermal treatment in catalysts derived from $\mathrm{Cu}-\mathrm{Al}$ hydrotalcites-like compounds in the NO reduction by CO, Catalysis Today, 2016.

FIGUEIREDO, J.L.; RIBEIRO, F.R.; Catálise Heterogênea, Fundação Calouste Gulbenkian, p.92-98, 1989;

LI, W.; WU, P.; ZHU, Y.; HUANG, Z.; LU, Y.; LI, Y.; DANG, Z.; ZHU, N. Catalytic degradation of bisphenol A by CoMnAl mixed metal oxides catalyzed peroxymonosulfate: Performance and mechanism. Chemical Engineering Journal, v.279, p. 93-102, 2015.

NIETO, J.M.L.; DEJOZ, A.; VAZQUEZ, M.I. Preparation, characterization and catalytic properties of vanadium oxides supported on calcined Mg/Al-hydrotalcite. Applied Catalysis A: General, v. 132, p. 41-59, 1995

PAULINO P.N.; ZACHARIAS M.A.; MONTEIRO W.R.; RODRIGUES J.A.J.; CORTEZ G.G. Propriedades catalíticas de catalisadores à base de óxido de vanádio suportado em alumina-nióbio In: CONGRESSO BRASILEIRO DE CATÁLISE, 14; 2007; Porto de Galinhas, -PE, Brasil. Anaais p.110.

SIM, J.S.; GONG, M.J., CHANG, K.S. Preliminary performance studies of Al-Co-Mn mixed oxide and Ag-doping por the purpose of PGM-free DOC. Applied Catalysis A: General, v.480, p. 120-127, 2014.

TEIXEIRA, V. G.; COUTINHO, F. M. B.; GOMES, A S.; Principais métodos de caracterização da porosidade de resinas à base de divinilbenzeno. Química Nova, v. 24, p. 808-818, 2001.

WU, M.; ZHAN, W.; GUO, Y.; WANG, Y.; GUO, Y.; GONG, X.; WANG, L.; LU, G. Solvent-free selective oxidation of cyclohexane with molecular oxygen over manganese oxides: Effect of the calcinations temperature. Chinese Journal of Catalysis, v. 37, p. 184192, 2016. 